\title{
Improved Methods for the Imputation of Missing Data by Nearest Neighbor Methods
}

Technical Report Number 172, 2014

Department of Statistics

University of Munich

http://www.stat.uni-muenchen.de 


\title{
Improved Methods for the Imputation of Missing Data by Nearest Neighbor Methods
}

\author{
Gerhard Tutz and Shahla Ramzan \\ Ludwig-Maximilians-Universität München \\ Akademiestraße 1, 80799 München
}

October 13, 2014

\begin{abstract}
Missing data is an important issue in almost all fields of quantitative research. A nonparametric procedure that has been shown to be useful is the nearest neighbor imputation method. We suggest a weighted nearest neighbor imputation method based on $L_{q}$-distances. The weighted method is shown to have smaller imputation error than available NN estimates. In addition we consider weighted neighbor imputation methods that use selected distances. The careful selection of distances that carry information on the missing values yields an imputation tool that outperforms competing nearest neighbor methods distinctly. Simulation studies show that the suggested weighted imputation with selection of distances provides the smallest imputation error, in particular when the number of predictors is large. In addition, the selected procedure is applied to real data from different fields.
\end{abstract}

Keywords: kernel function, weighted nearest neighbors, cross-validation, weighted imputation, MCAR,

\section{Introduction}

Missing data have always been a challenging topic for researchers. Ignoring all the missing cases is not a good strategy to deal with missing values. In the literature many techniques have been suggested since the 1980s to impute missing data, see, for example, Little and Rubin (1987), Schafer (2010). Broadly speaking, the methods for filling the incomplete data matrix can be divided into two main categories; single imputation and multiple imputation (Little and Rubin (1987)). A well known and computationally simple method for imputation of missing data is mean substitution. A disadvantage of the method is that the correlation structure among the predictors is ignored. An alternative is the nearest neighbors approach, which uses the observations in the neighborhood to impute missing values. Nearest neighbors as a nonparametric concept in discrimination dates back to Fix and Hodges (1951). The approach has been successfully used to impute data in gene expression (Troyanskaya et al. (2001), Atkeson et al. (1997), Hastie et al. (1999)), machine learning (Batista and Monard (2002)), medicine (Waljee et al. (2013)), forestry (Eskelson et al. (2009), Hudak et al. (2008)), and compositional data (Hron et al. (2010)) etc. 
An important aspect in missing data imputation is the pattern of missing values because the selection of an imputation procedure is determined by this pattern. Little and Rubin (1987) defined three categories of missing data; missing completely at random (MCAR), missing at random (MAR), and not missing at random (NMAR). Missing completely at random (MCAR) refers to the data in which the probability of a particular missing values does not depend on the variable itself or any other variable in the data set (Little and Rubin (1987), Allison (2001)). Most imputation methods assume the data to be at least MAR, if not MCAR, and so does the nearest neighbor method.

Hastie et al. (1999) proposed to use weights based on the Euclidean distance for the selection of the nearest neighbors. A comparison of $k$ nearest neighbor imputation (KNNimpute) with mean imputation and singular value decomposition (SVD) techniques for gene expression data was given by Troyanskaya et al. (2001). The results of their simulation studies showed that the method performs well when compared to mean imputation and singular value decomposition (SVD) approaches, see also Troyanskaya et al. (2003). In a comparative study of single imputation methods, Malarvizhi and Thanamani (2012) found that median or standard deviation substitution perform better than mean substitution. Therefore replacement of a missing value with simple average is not an efficient method. A further comparison of KNNimpute with mean, OLS and PLS imputation methods for imputation in microarray data was given by Nguyen et al. (2004). The findings of the study showed that KNNimpute performs well.

Several alternative procedures have been proposed that rely on the basic concept to impute values by building averages over qualifying neighbors, see, for example Ouyang et al. (2004), Kim et al. (2004), Sehgal et al. (2005), Scheel et al. (2005). Liew et al. (2011) and Moorthy et al. (2014) reviewed the available methods and algorithms for the imputation of missing values with a focus on gene expression data. Johansson and Hakkinen (2006) proposed WeNNI, which utilizes continuous weights in the nearest neighbors imputation procedure. B $\varnothing$ et al. (2004) and Wasito and Mirkin (2005) proposed a nearest neighbors procedure with a modification based on the least square principle. Other variants include local least squares (LLSimpute) by Kim et al. (2005), sequential local least square (SLLSimpute) by Zhang et al. (2008) and iterative local least square (ILLSimpute) by Cai et al. (2006).

A drawback of NN methods is that their performance depends on $k$. For example, KNNimpute typically performs well when $k$ is between 5 to 10 , but the performance deteriorates for larger $k$ (Yoon et al. (2007)). We propose a localized approach to missing data imputation that uses a weighted average of nearest neighbors using $L_{q}$ distances. For the high-dimensional case, we propose a new distance that explicitly uses the correlation among variables. The proposed method automatically selects the relevant variables that contribute to the distance and thus does not depend on $k$.

The paper is organized as follows: in Section 2 the $L_{q}$ distance is used to define a weighted imputation estimate. In a simulation study the weighted approach is compared to the unweighted approach. In Section 3, the weighted imputation with selection of predictors is introduced and compared to alternative imputation techniques. In Section 4 several applications to real data sets are given. 


\section{Weighted Neighbors}

When using nearest neighbors to impute data several choices have to be made. In particular one has to define what nearest neighbors are, that means, how they are computed and which distance measures are used. Then one has to choose how these nearest neighbors are used to obtain an imputed value. These choices to be made are considered in the following sections.

\subsection{Distances and Computation of Nearest Neighbors}

Let $n$ observations on $p$ covariates be collected. The corresponding $n \times p$ data matrix is given by $\boldsymbol{X}=\left(x_{i s}\right)$, where $x_{i s}$ denotes the $i$ th observation of the $s$ th variable. Let $\boldsymbol{O}=\left(o_{i s}\right)$ denote the corresponding $n \times p$ matrix of dummies with entries

$$
o_{i s}= \begin{cases}1 & \text { if } x_{i s} \text { was observed } \\ 0 & \text { for missing value. }\end{cases}
$$

Distances between two observations $\boldsymbol{x}_{i}$ and $\boldsymbol{x}_{j}$, which are represented by rows in the data matrix, can be computed by using the $L_{q}$-metric for the observed data. Then one uses the distances

$$
d_{q}\left(\boldsymbol{x}_{i}, \boldsymbol{x}_{j}\right)=\left[\frac{1}{m_{i j}} \sum_{s=1}^{p}\left|x_{i s}-x_{j s}\right|^{q} I\left(o_{i s}=1\right) I\left(o_{j s}=1\right)\right]^{1 / q},
$$

where $m_{i j}=\sum_{s=1}^{p} I\left(o_{i s}=1\right) I\left(o_{j s}=1\right)$ denotes the number of valid components in the computation of distances. The indicator function $I(a)$, which is used in the definition, has the value 1 , if $a$ is true and 0 otherwise. Thus, computation of distances does not use all the components of the vectors. It uses only those components for which observations in both vectors are available. The actually used components in the computation of neighbors is given by $C_{i j}=\left\{s: I\left(o_{i s}=\right.\right.$ 1) $\left.I\left(o_{j s}=1\right)=1\right\}$. The distances are used to define the nearest neighbors when imputing a specific value. It should be noted that the number of components that are used varies over the sample because $C_{i j}$ depends on the specific observation for which imputations are to be made.

Similar concepts to define distances and therefore nearest neighbors were used by Hastie et al. (1999), Troyanskaya et al. (2001), Myrtveit et al. (2001) and Kim et al. (2005). They used the Euclidean distance, $q=2$. Other alternatives to compute distances in gene expression studies were by use of the Pearson correlation (Dudoit et al. (2002), Bø et al. (2004)) and covariance estimate (Sehgal et al. (2005)).

\subsection{Imputation Procedure}

\section{Imputation for Fixed Number of Neighbors}

Let us consider imputation for $\boldsymbol{x}_{i}$ in component $s$, that is, $o_{i s}=0$. The imputation estimate for $\boldsymbol{x}_{i}$ is based on the $k$ nearest neighbors in the reduced data. One determines the $k$ nearest neighbors from the corresponding $(\tilde{n} \times p)$-dimensional reduced data set $\tilde{\boldsymbol{X}}=\left(x_{i j}, o_{i s}=1\right)$ obtaining

$$
\boldsymbol{x}_{(1)}, \ldots, \boldsymbol{x}_{(k)} \quad \text { with } \quad d\left(\boldsymbol{x}_{i}, \boldsymbol{x}_{(1)}\right) \leq \cdots \leq d\left(\boldsymbol{x}_{i}, \boldsymbol{x}_{(k)}\right),
$$

where $\boldsymbol{x}_{(j)}^{T}=\left(x_{(j) 1}, \ldots, x_{(j) p}\right)$ denotes the $j$ th nearest neighbor. Then the $i m$ putation value for fixed $k$ is 


$$
\hat{x}_{i s}=\frac{1}{k} \sum_{j=1}^{k} x_{(j) s}
$$

Thus the missing value in the sth component of observation vector $\boldsymbol{x}_{i}$ is replaced by the average of the corresponding values of the $k$ nearest neighbors. The accuracy of the method is mainly determined by the number of neighbors that are used. Simple rules use a fixed number of neighbors that are chosen by the experimenter. However, it is advantageous to consider the number of neighbors as a tuning parameter that is chosen in a data-driven way, for example by cross-validation (see next section).

\section{Imputation by Weighting}

A disadvantage of the imputation based on the $k$ nearest neighbors is that the value of the first nearest neighbor has the same importance as the $k$ th nearest neighbor. A more appropriate method uses weights that account for the distance of the observations. We consider a weighted average of neighbors based on distances that are determined by kernel functions. The weighted imputation estimate considered here has the form

$$
\hat{x}_{i s}=\sum_{j=1}^{k} w\left(\boldsymbol{x}_{i}, \boldsymbol{x}_{(j)}\right) x_{(j) s}
$$

with weights

$$
w\left(\boldsymbol{x}_{i}, \boldsymbol{x}_{j}\right)=K\left(d\left(\boldsymbol{x}_{i}, \boldsymbol{x}_{j}\right) / \lambda\right) / \sum_{l=1}^{k} K\left(d\left(\boldsymbol{x}_{i}, \boldsymbol{x}_{j}\right) / \lambda\right),
$$

where $K($.$) is a kernel function (for example tricube, Gaussian) and \lambda$ is a tuning parameter. For small $\lambda$, the weights are decreasing very strongly with the distance, whereas for $\lambda \rightarrow \infty$, one uses equal weights for all neighbors. To make $\lambda$ the crucial tuning parameter one should use a large number of potential neighbors. In the extreme case, if one uses $k=\tilde{n}$, the window-width $\lambda$ is the only tuning parameter.

Alternative weights were used by Troyanskaya et al. (2001). They used the weighted average over the $k$ nearest neighbors based on the Euclidean distance with the weights determined by the inverse of the Euclidean distance.

\subsection{Choice of Tuning Parameters by Cross-validation}

An important issue in weighted nearest neighbors imputation techniques is the selection of the tuning parameter $\lambda$. One option is to use the same method that is in common use when selecting the optimal number of nearest neighbors $k$, namely cross-validation, see, for example,Dudoit et al. (2002). Also some $\mathrm{R}$ packages provide cross-validation as a tool to choose the optimal number of nearest neighbors (Waljee et al. (2013)). The concept is to artificially delete some values in the data matrix and compute the mean squared error (MSE) or mean absolute error (MAE) for the artificially deleted data. Then one chooses the value for which MSE or MAE takes the minimal value.

In our approach we generate completely at random (MCAR) $m^{*}$ artificially missing values from the available data $\left\{x_{i s}: o_{i s}=1\right\}$. Let $\boldsymbol{X}^{*}$ denote the $n \times p$ data matrix containing the originally missing values $\left(\left\{x_{i s}: o_{i s}=0\right\}\right)$ and the 
artificially missing values $\left(\left\{x_{i s}^{*}: o_{i s}^{*}=0\right\}\right)$. Then the mean absolute error (MAE) for these observations is defined by

$$
\operatorname{MAE}\left(\boldsymbol{X}^{*}\right)=\frac{1}{m^{*}} \sum_{x_{i s}: O_{i s}^{*}=0}\left|x_{i s}^{*}-x_{i s(\text { imputed })}^{*}\right| .
$$

This procedure is repeated $R$ times yielding the averaged value

$$
\operatorname{MAE}_{\mathrm{CV}}=\frac{1}{R} \sum_{r=1}^{R} \operatorname{MAE}\left(\boldsymbol{X}_{r}^{*}\right)
$$

where $\boldsymbol{X}_{r}^{*}$ denotes the matrix with both types of missing values in the $r$ th replication. In the same way the cross-validated mean square error (MSE) is computed by using

$$
\operatorname{MSE}\left(\boldsymbol{X}^{*}\right)=\frac{1}{m^{*}} \sum_{x_{i s}: o_{i s}^{*}=0}\left(x_{i s}^{*}-x_{i s(\text { imputed })}^{*}\right)^{2}
$$

yielding $\mathrm{MSE}_{\mathrm{CV}}$.

The cross-validation is done in the following way:

1. For a specific value of $\lambda$;

a. Artificially delete $m^{*}$ value/s in the data matrix.

b. Impute these missing values and calculate MSE or MAE.

c. Repeat a and b, $R$ times for example, $R=100$ depending upon data, to find $\mathrm{MSE}_{\mathrm{CV}}$ or $\mathrm{MAE}_{\mathrm{CV}}$.

2. Repeat steps a-c for all values of $\lambda$, and choose the one with minimum $\mathrm{MSE}_{\mathrm{CV}}$ or $\mathrm{MAE}_{\mathrm{CV}}$ as optimal $\lambda$.

It is unclear how many values should be deleted artificially for the purpose of cross-validation. We used several values ranging from the deletion of only one observation to $10 \%$ of the data matrix. In our simulations the resulting values of $\lambda$ were very similar. Therefore we proceed with the deletion of $10 \%$ of the data matrix.

\subsection{Performance Measures}

If the tuning parameter has been chosen the performance of the different imputation methods is compared on the basis of the mean squared error (MSE) and the mean absolute error (MAE) computed from the original and imputed values in the same way as in cross-validation (Troyanskaya et al. (2001), Junninen et al. (2004), Hudak et al. (2008)). In simulation studies evaluation is easy. If $\left\{x_{i s}: o_{i s}=0\right\}$ are the missing values in the data matrix $\boldsymbol{X}$, one computes the corresponding imputed values using the $\lambda$ selected by cross-validation in Section 2.3. The performance is by measured by computing MAE or MSE by using the missing observations and the imputed values.

When using real data the original values are not known. Then, performance is evaluated by considering observations with $\left\{x_{i s}: o_{i s}=1\right\}$ as missing and computing the corresponding imputed value to obtain MAE or MSE. The number of values that are considered as missing in the evaluation of the performance depends upon the data. 


\subsection{Evaluation of Weighted Neighbors}

The correlation structure of the data plays an important role in the selection of the imputation method, see, for example Feten et al. (2005). Therefore, in this section we investigate the dependence of the method on the correlation between variables and compare the un-weighted version with the weighted nearest neighbor version of imputation in a simulation study.

\section{Imputation Using Fixed Number of Nearest Neighbors}

In the simulated data, variables are drawn from a $p$-dimensional multivariate normal distribution with mean $\mathbf{0}$ and the correlation among the covariates (among the columns of design matrix) is $\rho$. Five values of $\rho=0.1,0.3,0.5,0.7$, and 0.9 were used. For each value of $\rho, S=50$ samples, each of size $n=50$, were drawn for $p=10$ covariates from $N(\mathbf{0}, \boldsymbol{\Sigma})$, where $\boldsymbol{\Sigma}$ is a correlation matrix with pairwise correlations $\rho$ among the covariates. The unweighted nearest neighbor is used to impute (i) $5 \%$, and (ii) $25 \%$ missing values (MCAR) in each simulation setting. The number of nearest neighbors $k \in\{1, \ldots, 40\}$, were used to estimate the missing values. The performance of imputation in terms of mean squared error (MSE) and mean absolute error (MAE) is shown in Fig. 1 for different values of $k$ and for selected values of $\rho(0.3,0.5,0.9)$. Figure 1 shows that for low correlation, for example, $\rho=0.3$, the MSE/MAE is high for small values of $k$ and reduces with increasing value of $k$ but remains almost stable for $k \geq 10$. If one excludes small values of $k$, the performance does not strongly depend on the chosen number of the next neighbors. The picture changes if the correlation among the variables is stronger. Then there is a distinct minimum and the performance deteriorates if $k$ increases. It is, in particular, noteworthy that the performance is much better when variables are correlated. For $\rho=0.3$, $5 \%$ missing, the best obtained value is about 0.7 whereas for $\rho=0.9,5 \%$ missing, the best value is below 0.2 . Imputation works much better if variables are correlated because only then information from the other variables is available. Therefore, we will focus on correlated data in the following.

\section{Weighted Imputation}

In the next simulation the weighted nearest neighbor method, henceforth $w N N$, is compared to un-weighted imputation. Again $S=50$ samples each of size $n=50$ were drawn for $p=15$ covariates from $N(\mathbf{0}, \boldsymbol{\Sigma})$. The values of pairwise correlation used in $\boldsymbol{\Sigma}$ are $\rho=0.5,0.7$, and 0.9 . We considered $10 \%$ and $25 \%$ of the total values as missing completely at random in each sample. The distance (1) with $q=1$ ( $L_{1}$ distance) and $q=2$ ( $L_{2}$ or Euclidean distance) were used to impute the missing data. The optimal value of tuning parameter $\lambda$ for weights (4) was chosen on the basis of MSE (Mean Squared Error) and MAE (Mean Absolute Error) by cross-validation procedure. The mean squared error and the mean absolute error showed similar behavior, so the results of MSE only are presented. Weights were calculated by using a Gaussian kernel because simulations had shown that the performance for the Gaussian kernel was slightly better than for alternative kernels like the triangular kernel.

Fig. 2 shows the MSEs for the nearest neighbor imputation method with fixed values of $k=5,10,20,40$, for $k$ chosen by cross-validation, and for the weighted approach with $k=20,40$ as the maximal neighbors. The boxplots of MSE with $L_{1}$ distance are shown in the upper panel of Fig. 2, and $L_{2}$ distance in the lower panel. It is seen that $w N N$ imputation works well when the data 

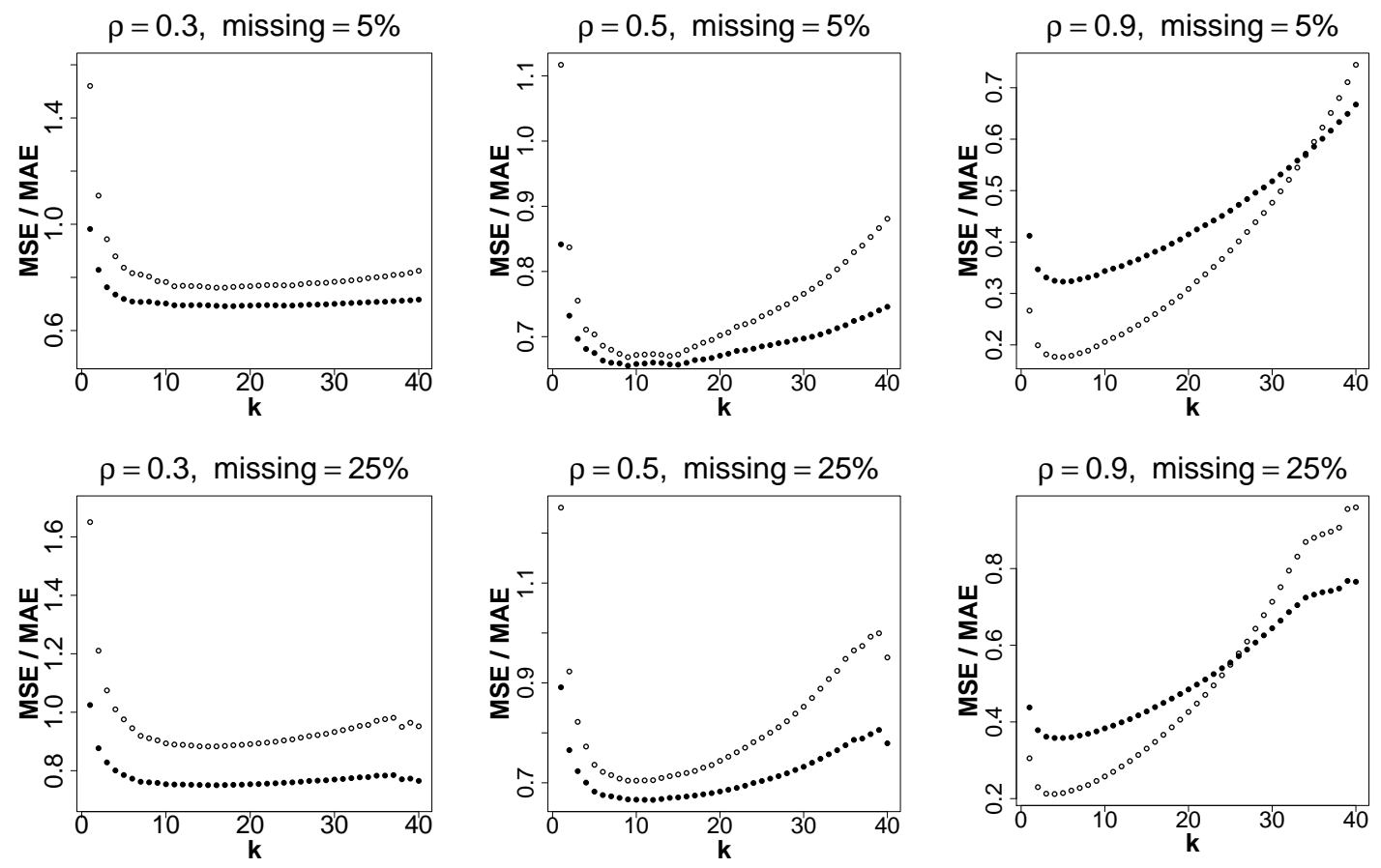

Figure 1: Illustration of simulation study: Mean Squared Error (MSE) and Mean Absolute error (MAE) for unweighted nearest neighbors using $L_{2}$ metric for fixed values of $k$ (number of nearest neighbors), with $5 \%$ missing data (Upper panel) and $25 \%$ missing data (lower panel). Circles represent MSE and solid circles represent MAE.

are highly correlated. The MSE for $\rho=0.5,10 \%$ missing, is larger than 0.6, whereas for $\rho=0.9,10 \%$ missing, it is below 0.2 (Fig. 2(a)). Similar results are found for the $L_{2}$ distance (Fig. 2(b)). Overall, $L_{1}$ and $L_{2}$ distances produce nearly the same mean square errors in all the data settings.

In addition to the dependence on the correlation structure one objective of the simulation study is to investigate if cross-validation is able to find a proper value for $k$, the number of nearest neighbors to be used. The boxplots show that, although some specific values of $k$ yield better performance, the procedure that selects $k$ by cross-validation (denoted by optimal $k$ ) does fairly well. The other point of interest is how the proposed weighted imputation performs. It is seen that weighted imputation $(w N N)$ always performs better than imputation for fixed value of $k$, when the window width and the number of nearest neighbors, respectively, are chosen by cross-validation. The detailed results are shown in Table 1, where for each scenario the method that performed best is shown in boldface. It is seen that there is not much difference when allowing for 20 or 40 nearest neighbors in the weighting procedure. Nevertheless, for 40 the performance tends to be slightly better. In summary, weighted imputation turns out to be an attractive alternative that is computationally not more intensive than imputation with fixed $k$. 

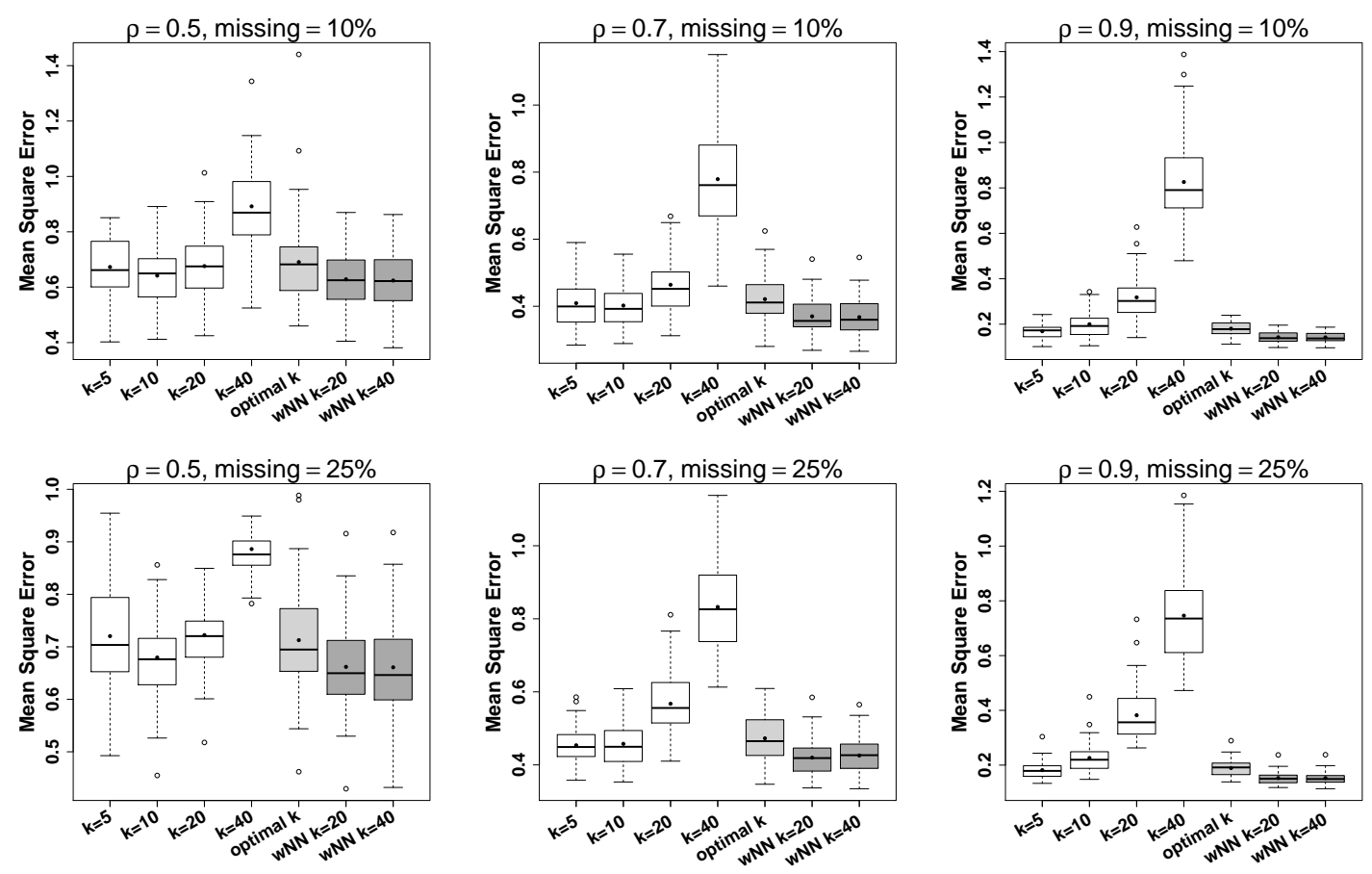

(a) $L_{1}$ distance
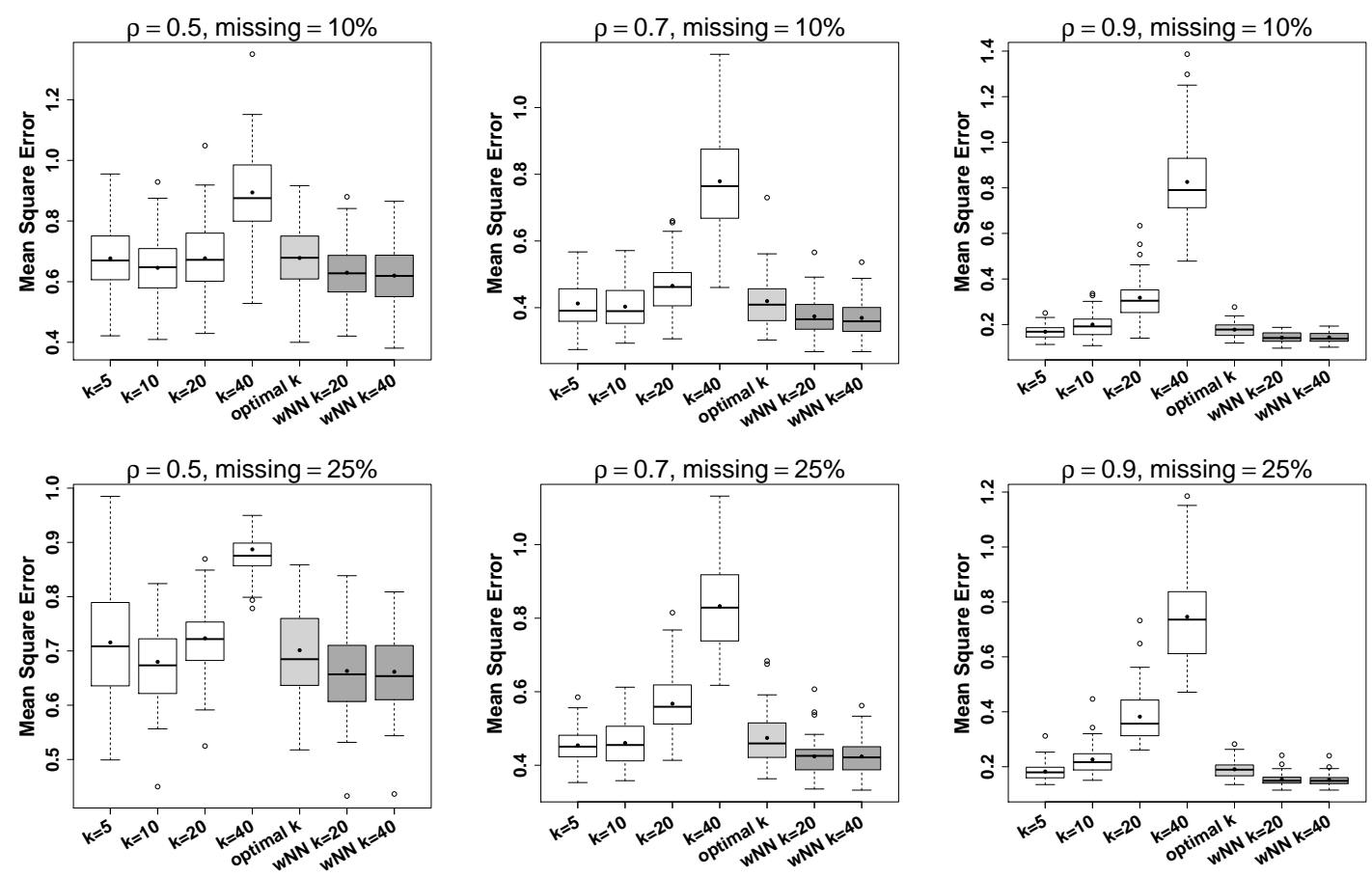

(b) $L_{2}$ distance

Figure 2: Boxplots of Mean Squared Error for NN Imputation using $L_{1}$ distance (a) and $L_{2}$ distance (b). Imputation is done by using unweighted/fixed values of $k$ $=5,10,20,40$ nearest neighbors (white boxes), for optimal value of $k$ selected by cross-validation (light grey boxes), and weighted approach $(w N N)$ with maximum $k$ $=20,40$ using Gaussian kernel. Solid circles within boxes show the mean values. 
TABLE 1: MSE for NN imputation with weighted and unweighted approach using $L_{1}$ and $L_{2}$ distances

\begin{tabular}{|c|c|c|c|c|c|c|c|c|}
\hline \multirow[b]{3}{*}{$\rho$} & \multirow[b]{3}{*}{ missing (\%) } & \multicolumn{7}{|c|}{$L_{1}$ distance } \\
\hline & & \multicolumn{5}{|c|}{ Fixed / unweighted } & \multicolumn{2}{|c|}{ wNN } \\
\hline & & $k=5$ & $k=10$ & $k=20$ & $k=40$ & optimal $k$ & $k=20$ & $k=40$ \\
\hline 0.5 & 10 & 0.6729 & 0.6419 & 0.6761 & 0.8920 & 0.6907 & 0.6286 & 0.6242 \\
\hline & 25 & 0.7203 & 0.6796 & 0.7223 & 0.8861 & 0.7127 & 0.6618 & 0.6609 \\
\hline 0.7 & 10 & 0.4093 & 0.4021 & 0.4642 & 0.7790 & 0.4213 & 0.3700 & 0.3675 \\
\hline & 25 & 0.4537 & 0.4578 & 0.5674 & 0.8323 & 0.4728 & 0.4199 & 0.4258 \\
\hline \multirow[t]{5}{*}{0.9} & 10 & 0.1689 & 0.1999 & 0.3181 & 0.8259 & 0.1803 & 0.1424 & 0.1418 \\
\hline & 25 & 0.1817 & 0.2261 & 0.3822 & 0.7459 & 0.1893 & 0.1528 & 0.1527 \\
\hline & & \multicolumn{7}{|c|}{$L_{2}$ distance } \\
\hline & & \multicolumn{5}{|c|}{ Fixed / unweighted } & \multicolumn{2}{|c|}{$\mathrm{wNN}$} \\
\hline & & $k=5$ & $k=10$ & $k=20$ & $k=40$ & optimal $k$ & $k=20$ & $k=40$ \\
\hline \multirow[t]{2}{*}{0.5} & 10 & 0.6768 & 0.6459 & 0.6772 & 0.8942 & 0.6781 & 0.6298 & 0.6200 \\
\hline & 25 & 0.7157 & 0.6799 & 0.7234 & 0.8871 & 0.7013 & 0.663 & 0.6616 \\
\hline \multirow[t]{2}{*}{0.7} & 10 & 0.4126 & 0.4032 & 0.4655 & 0.7792 & 0.4197 & 0.3741 & 0.3694 \\
\hline & 25 & 0.4541 & 0.4606 & 0.5674 & 0.8326 & 0.4743 & 0.4240 & 0.4239 \\
\hline \multirow[t]{2}{*}{0.9} & 10 & 0.1685 & 0.2002 & 0.3181 & 0.8259 & 0.1779 & 0.1426 & 0.1434 \\
\hline & 25 & 0.1827 & 0.2263 & 0.3823 & 0.7459 & 0.1908 & 0.1536 & 0.1527 \\
\hline
\end{tabular}

\section{Weighted Neighbors Including Selection of Predictors}

\subsection{Selection of Dimensions}

Traditionally the distances are computed from all the available components of the observations. However, as will be shown, in high-dimensional settings imputation suffers from the curse of dimensionality. Therefore, we propose to compute distances from selected dimensions only. Since imputation is successful in particular when the predictors are highly correlated the selection of the dimensions will be linked to the correlation between predictors. In the following an extended version of the weighted nearest neighbor imputation method is given that uses only the correlated predictors for the computation of the distances.

Let us consider imputation for $\boldsymbol{x}_{i}$ in component $s\left(o_{i s}=0\right)$. When computing distances from the reduced data set $\left\{\boldsymbol{x}_{j}, o_{j s}=1\right\}$, we use an additional weight in the distances. More concrete, for $L_{q^{-}}$distance one computes component-specific distances by

$$
d_{q, C}\left(\boldsymbol{x}_{i}, \boldsymbol{x}_{j}\right)=\left\{\frac{1}{m_{i j}} \sum_{l=1}^{p}\left|x_{i l}-x_{j l}\right| I\left(o_{i s}=1\right) I\left(o_{j s}=1\right) C\left(r_{s l}\right)\right\}^{1 / q},
$$

where $r_{s l}$ is the empirical correlation between covariates $s, l$ and $C($.$) is a convex$ function defined on the interval $[-1,1]$ that transforms the correlations into weights. The transformation is constructed such that covariates that are strongly correlated with component $s$ strongly contribute to the computation of distances, while components that are not correlated do not contribute to the distance.

One convex function $C($.) that can be used is defined by

$$
C(r)= \begin{cases}\frac{|r|}{1-c}-\frac{c}{1-c} & \text { if }|r|>c \\ 0 & \text { if }|r| \leq c .\end{cases}
$$


It is linear in the absolute value of the correlation. If $\left|r_{s j}\right| \leq c$, the component $s$ does not contribute to the distance. Weights increase linearly with increasing correlation and have weight 1 if $|r|=1$. The threshold parameter $c$ can be chosen as fixed, for example, $c=0$ or be considered as an additional tuning parameter. A smoother function, which will also be used, is the power function

$$
C(r)=|r|^{m},
$$

with $m$ as additional tuning parameter. One problem is that $r_{s l}$ cannot be computed since missing values are present in the data. Therefore we use a simple first step imputation by use of un-weighted five nearest neighbors and compute the correlations from the observed and imputed data. Then the actual imputation is carried out by using the correlations computed in the first step.

The weighted $L_{q}$ distances with selection of dimensions includes the calculation of an additional tuning parameter. In addition to the tuning parameters for the kernels, $\lambda$, one has to find an appropriate value $c$ for the convex function (8) or an integer $m$ for the convex function (9). The parameters are chosen in the same way as $\lambda$ in Section 2.3, namely by cross-validation. For the simultaneous selection of the tuning parameters $(\lambda, c)$ or $(\lambda, m)$ cross-validation is computed on on a two-dimensional grid of values. We will refer to this method as $w N N S$ elect. We will characterize the linear function by $c$, and the power function by the parameter $m$.

\subsection{Evaluation of the Selected Weighted Neighbors}

The simulation study in Section 2.5 showed that for highly correlated predictors weighted nearest neighbors $(w N N)$ perform better than unweighted distances. Moreover, the results showed that $L_{1}$ and $L_{2}$ distances have very similar performance. In this section, the performance of the weighted approach with selection of predictors is investigated. We consider, in particular, two structures of the correlation matrix, blockwise correlation and autoregressive (AR) type correlation.

\section{Blockwise Correlation Structure}

Let the data matrix $\mathbf{X}_{(n \times p)}$, be partitioned into $\mathbf{X}=\left[\mathbf{X}^{(1)}, \mathbf{X}^{(2)}, \mathbf{X}^{(3)}\right]$ such that each element of $\mathbf{X}^{(q)}=\left[\mathbf{x}_{1}, \cdots, \mathbf{x}_{p_{q}}\right], q=1,2,3$, is a $n \times 1$ random vector. The partitioned correlation matrix has the form

$$
\Sigma=\left(\begin{array}{ccccc}
\Sigma_{11} & \vdots & \Sigma_{12} & \vdots & \Sigma_{13} \\
\cdots & \cdots & \cdots & \cdots & \cdots \\
\Sigma_{21} & \vdots & \Sigma_{22} & \vdots & \Sigma_{23} \\
\cdots & \cdots & \cdots & \cdots & \cdots \\
\Sigma_{31} & \vdots & \Sigma_{32} & \vdots & \Sigma_{33}
\end{array}\right),
$$

where $\Sigma_{i i}$, is the matrix containing pairwise correlations $\rho$ of the elements of $\mathbf{X}^{(q)}, q=1,2,3$, that is, all components have (within) correlation $\left(\rho_{w}\right)$. The matrix $\Sigma_{i j}$, contains the pairwise (between) correlations $\left(\rho_{b}\right)$ between all the elements of $\mathbf{X}^{(q)}$ and $\mathbf{X}^{(r)}$, for $i, j=1,2,3 \forall i \neq j$. 


\section{AR-Type Correlation Structure}

The second correlation structure we consider is the exponential or autoregressive type correlation structure. An autoregressive of order 1 correlation matrix is defined by

$$
\Sigma=\left(\rho^{|i-j|}\right),
$$

where $i, j=1, \ldots, p$ and $\rho$ is the pairwise correlation between predictors and $p$ is the number of predictors or variables in the data matrix.

\section{Comparison of Fixed and Selected Values of Tuning Parameters}

In the weighted imputation method considered in Section $2(w N N)$, one tuning parameter $\lambda$ for the kernel weights had to be chosen. The method with selection of predictors (7) involves one additional tuning parameter, $c$ or $m$. In the following we first investigate if the data driven selection of the tuning parameters $(\lambda, c)$ or $(\lambda, m)$ by cross-validation works.

We generated $S=50$ samples of size $n=50$ for $p=30$ predictors drawn from a multivariate normal distribution with $N(\mathbf{0}, \boldsymbol{\Sigma})$. The correlation matrix $\Sigma$ had either blockwise or autoregressive type structure. The data settings used for the simulations were

\begin{tabular}{cllc}
\hline Setting & Structure & Correlation & missing \\
\hline 1 & Blockwise & $\rho_{w}=0.7, \rho_{b}=0.1$ & $10 \%$ \\
2 & & & $25 \%$ \\
3 & & $\rho_{w}=0.9, \rho_{b}=0.1$ & $10 \%$ \\
4 & & & $25 \%$ \\
5 & Autoregressive & $\rho=0.9$ & $10 \%$ \\
6 & & & $25 \%$ \\
\hline
\end{tabular}

In the first type of $\boldsymbol{\Sigma}$, the predictors were chosen in three blocks of 10 predictors each, that is, the $p \times 1$ random vector $\mathbf{X}=\left[\mathbf{X}^{(1)}, \mathbf{X}^{(2)}, \mathbf{X}^{(3)}\right]$, is such that $\mathbf{X}^{(1)}=\left[X_{1}, \ldots, X_{10}\right], \mathbf{X}^{(2)}=\left[X_{11}, \ldots, X_{20}\right]$, and $\mathbf{X}^{(3)}=\left[X_{21}, \ldots, X_{30}\right]$, where each element of $\mathbf{X}^{(q)},(q=1,2,3)$ is a random variable. The variables within each block were strongly correlated with $\rho_{w}=0.70,0.90$, but nearly uncorrelated with the variables in the other blocks with $\rho_{b}=0.10$, for $i, j=1,2,3 \forall i \neq j$. The second structure of $\boldsymbol{\Sigma}$ considered is the autoregressive type of order 1 with $\rho=0.9$. In each sample, $10 \%$ and $25 \%$ of the total values were replaced by missing values completely at random (MCAR).

In the simulation study, we used both convex functions (8) and (9) at fixed levels and selected by cross-validation. For tuning parameter $c$ in the linear function (8) we considered $c \in\{0.2,0.3,0.4,0.5\}$. Therefore, all the predictors whose correlation with the $s^{\text {th }}$ predictors was less than or equal to specified $c$, were discarded during the computation of distance. For the power function (9), we used three values of the tuning parameter, $m \in\{2,4,6\}$. The number of nearest neighbors $k$ was set to the maximum available neighbors. The large value of $k$ was chosen as the imputation procedure automatically chooses only relevant neighbors for the calculation of the distance. For weighted NN imputation with selection of predictors ( $w N N$ Select), cross-validation was used to compute MSE for the selection of optimal pair of the tuning parameters. For the weighted NN 

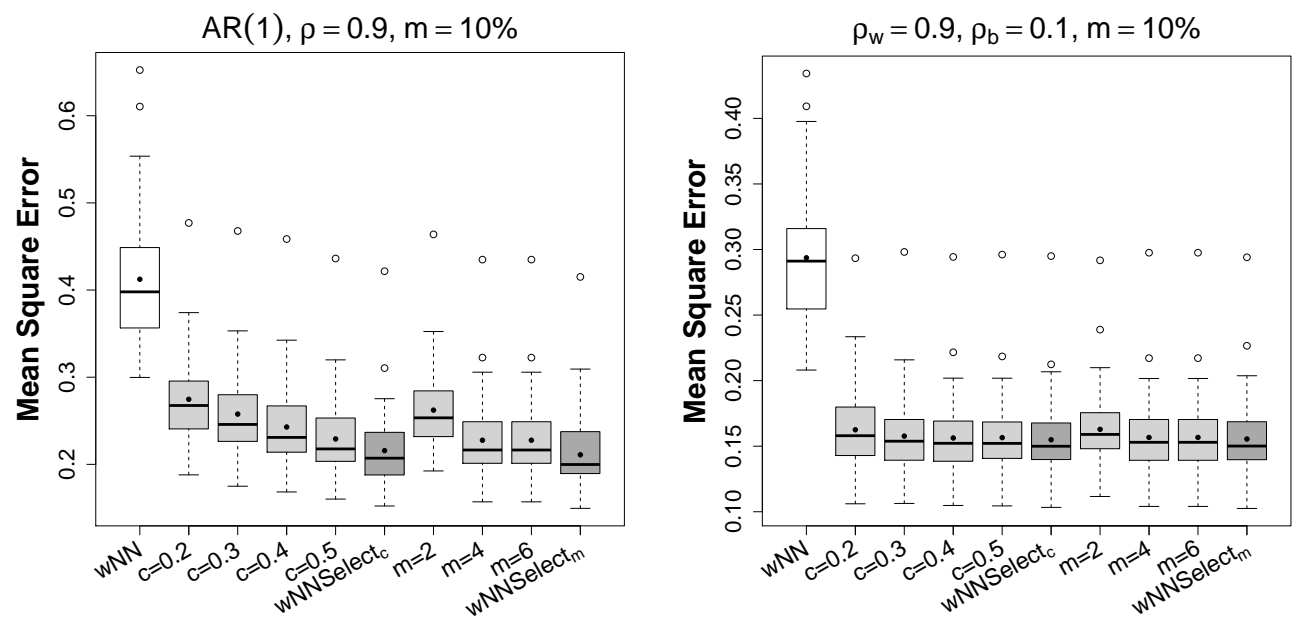

Figure 3: Comparison of weighted imputation (white boxes), weighted with selection of predictors at fixed $c=0.2,0.3,0.4,0.5 ; m=2,4,6$ (light grey boxes) and with values of tuning parameters chosen by cross-validation ( $w N N$ Select $_{c}$ and wNNSelect $_{m}$, dark grey boxes) based on $L_{2}$ distance in Autoregressive (left) and blockwise (right) correlation structure. Solid circles within boxes show the mean values.

imputation without selection of predictors $(w N N)$ the optimal $\lambda$ was also chosen on the basis of minimum MSE.

A visual comparison of weighted $(w N N)$ and weighted with selection of predictors approaches for fixed and the selected values of tuning parameter of convex functions $c$ and $m$ is given in Figure 3. Obviously, the method with selection of predictors ( $w N N$ Select) performs much better than simple weighted NN imputation without selection of distances $(w N N)$. Again the results from $L_{1}$ and $L_{2}$ distances do not differ significantly. It is also seen that choice of tuning parameters $c$ and $m$ works well. For both type of correlation matrices it provides smaller MSE than for fixed $c$ and $m$. Similar results were obtained for other simulation settings not shown here. Therefore we will proceed with the selected tuning parameter by double grid cross-validation.

\section{Dependence on the Number of Predictors}

Figure 3 already showed that the selection of sub spaces may yield better results. In the following we want to investigate how strong imputation based on distances computed from the whole set of predictors suffers when the number of predictors increases. Based on the results of the simulation study in the previous subsection we use cross-validation to select tuning parameters on a double grid $(\lambda$, $c)$ and $(\lambda, m)$.

We use $S=50$ sample of size $n=50$ with number of predictors $p \in\{5,10,20,30,40,50,60,70,80,90,100\}$ and $n=100$ with $p \in$ $\{5,10,20,30,40,50,60,70,80,90,100,120,150\}$ from $N(\mathbf{0}, \boldsymbol{\Sigma})$, where $\boldsymbol{\Sigma}$ is the $\mathrm{AR}(1)$ correlation matrix with $\rho=0.9$.

For comparison we use established nearest neighborhood methods that are available in the $\mathrm{R}$ environment ( $\mathrm{R}$ Core Team (2013)). The package impute from Bioconductor uses nearest neighbors to impute the missing expression values of continuous variables (Hastie et al. (2013)). The function kNN in R package VIM (Templ et al. (2013)) deals with data consisting of continuous, count, binary and categorical variables. By default, it uses Gower distance (Gower (1971)) for 
the computation of distances, but other metrics can also be used. Waljee et al. (2013) also used this package in their studies on clinical data. Another package imputation (Wong (2013)) is available that imputes the missing data using the algorithm provided by Troyanskaya et al. (2001). But the package sometimes does not yield estimates of all the missing data and such values are replaced by zeros by default.

Therefore, we chose the impute function from Bioconductor (Hastie et al.) and the kNN function from package VIM (Templ et al. (2013)) as benchmarks (shown as BIO and VIM respectively in Figure 5). Both methods use a predefined or fixed number of nearest neighbors $(k)$. To obtain comparable results we selected the number of nearest neighbors $(k)$ by using the same cross-validation algorithm as discussed in Section 2.3. For weighted imputation without selection of variables, the tuning parameter $\lambda$ was chosen using cross-validation with $m *$ corresponding to $10 \%$ and $R=10$. The values of the tuning parameters $(\lambda, c)$ and $(\lambda, m)$ were chosen via cross-validation on a double grid with $m *$ corresponding to $10 \%$ and $R=10$ for weighted imputation including selection of predictors.
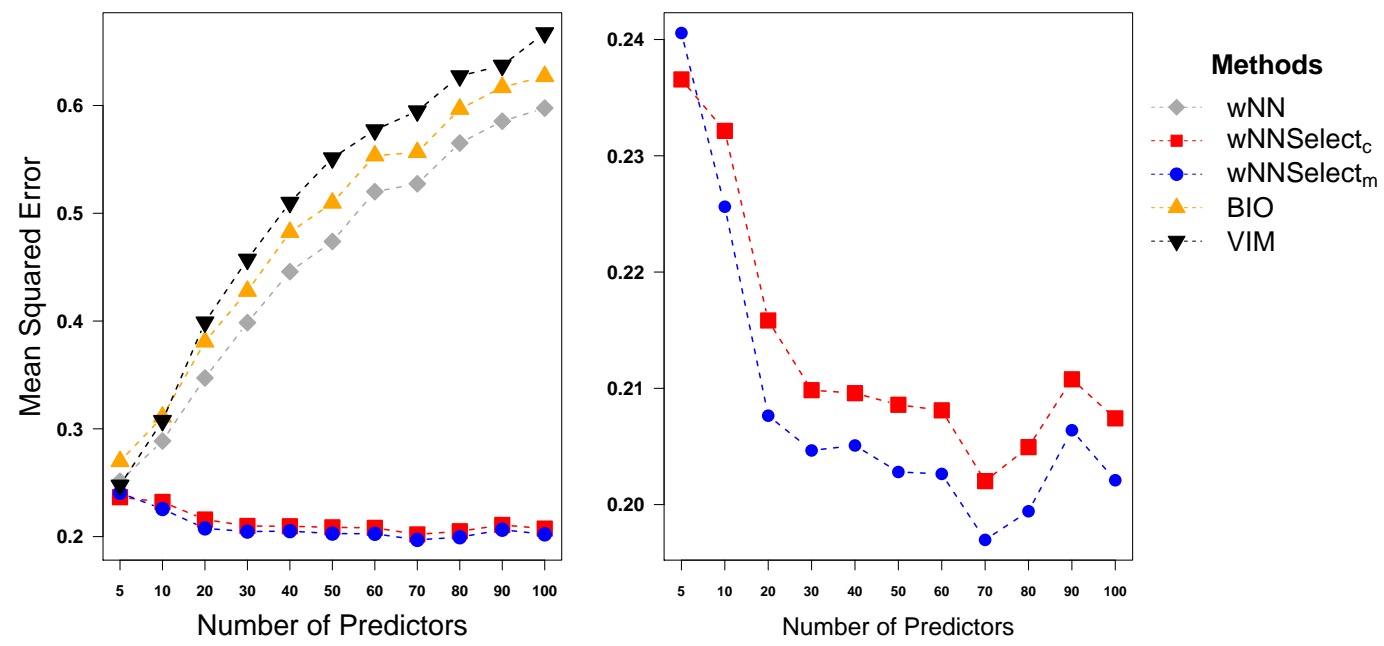

Figure 4: Comparison of average MSEs at different number of predictors, $n=50$ (upper panel) and $n=100$ (lower panel) using $A R(1)$ correlation matrix with $\rho=0.9$

Fig. 4(left panel) shows the average MSEs for the imputation methods under consideration. It is seen that all imputation methods that use all the predictors show poor performance for increasing numbers of predictors. Although more information is available because more predictors are observed the performance deteriorates. In contrast, the methods that allow for selection of predictors can use the additional information. They perform better if the number of predictors increases. It also should be noted that with increasing number of predictors, the proposed weighting function (without selection) performs even better. Fig. 4(right panel) shows the performance of these methods in a separate panel. It is seen that, in particular, the convex function (9) yields good results in terms of mean squared errors even when the number of predictors is large. 


\section{Comparison of Weighted Imputation and Benchmarks}

In the following we investigate more systematically the performance of the new methods ( $w N N$ and $w N N$ Select) and benchmark methods.

We consider $S=50$ samples of size $n=50,100$ from $N(\mathbf{0}, \boldsymbol{\Sigma})$, where $\boldsymbol{\Sigma}$ is the correlation matrix of blockwise or autoregressive structure. The simulation settings are as follows:

\begin{tabular}{clccl}
\hline Setting & Structure & $n$ & $p$ & Correlation \\
\hline 1 & Blockwise & 50 & 30 & $\rho_{b}=0.9, \rho_{w}=0.1$ \\
2 & & 100 & 45 & \\
3 & Autoregressive & 50 & 30 & $\rho=0.9$ \\
4 & & 100 & 40 & \\
\hline
\end{tabular}

For blockwise correlation with $n=50$ (setting 1 ), in $\boldsymbol{\Sigma}$, the predictors were chosen in three blocks of 10 predictors each, that is, the $p \times 1$ random vector $\mathbf{X}=\left[\mathbf{X}^{(1)}, \mathbf{X}^{(2)}, \mathbf{X}^{(3)}\right]$, is such that $\mathbf{X}^{(1)}=\left[X_{1}, \ldots, X_{10}\right], \mathbf{X}^{(2)}=$ $\left[X_{11}, \ldots, X_{20}\right]$, and $\mathbf{X}^{(3)}=\left[X_{21}, \ldots, X_{30}\right]$, where each element of $\mathbf{X}^{(q)}, \quad(q=$ $1,2,3)$ is a random variable. The variables within each block were strongly correlated with $\rho_{w}=0.90$, but nearly uncorrelated with the variables in the other blocks with $\rho_{b}=0.10$, for $i, j=1,2,3 \forall i \neq j$. Similarly, for $n=100$ (setting 2), the predictors were chosen in three blocks of 15 predictors each, that is, the $p \times 1$ random vector $\mathbf{X}=\left[\mathbf{X}^{(1)}, \mathbf{X}^{(2)}, \mathbf{X}^{(3)}\right]$, is such that $\mathbf{X}^{(1)}=\left[X_{1}, \ldots, X_{15}\right], \mathbf{X}^{(2)}=\left[X_{16}, \ldots, X_{30}\right]$, and $\mathbf{X}^{(3)}=\left[X_{31}, \ldots, X_{45}\right]$, where each element of $\mathbf{X}^{(q)}$, $(\mathrm{q}=1,2,3)$ is a random variable. In setting 3 and 4 , the autoregressive type $\boldsymbol{\Sigma}$ is of order 1 with $\rho=0.9$.

The percentage of missing values in each data setting was set to $1 \%, 5 \%, 10 \%, 20 \%, 25 \%$ and $30 \%$ of the total values in design matrix completely at random (MCAR). The tuning parameters for the proposed weighting function $w N N$ and $w N N$ Select were chosen by cross-validation with $m *=10 \%$. For the benchmark methods, the number of nearest neighbors $(k)$ was also chosen by use of the same cross-validation algorithm.

Figure 5 compares the proposed weighted imputation methods with the existing methods under Setting 1 and 3 at 5\%,15\%, and 25\% missing data. For the blockwise correlation structure (Fig. 5: upper panel for data setting 1), the weighting functions using $L_{2}$ metric with selection (shown as $w N N$ Select $_{c}$ and $w N N$ Select $t_{m}$ ) and without selection of predictors (shown as $w N N S$ ) provides smaller MSE than the benchmarks (shown as BIO and VIM). The best MSE is given by weighted selection of predictors (shown as $w N N$ Select $_{c}$ and $w N N$ Select $t_{m}$ ). The convex functions (linear (8) and power (9)) used for the selection of predictors provide nearly the same MSEs. For the autoregressive type correlation structure, (Fig. 5: lower panel for data Setting 3), at 5\% missing the best MSE is obtained near 0.1 , at $15 \%$ missing is near 0.2 and at $25 \%$ missing MSE is near 0.3. Therefore, in autoregressive type correlation the average MSE increases with an increase in the missing values. Similar results were obtained for Settings 2 and 4 with remaining missing percentages, see Table 2. In each data setting the value of smallest MSE is shown as boldfaced.

As seen from Table 2 for blockwise correlation with $n=50$ (upper left panel), at $1 \%$ missing data the smallest MSE was obtained by weighted selection of predictors $w N N$ Select $_{m}, 0.1338$. This value is much smaller than obtained by existing NN imputation methods ( 0.2846 by BIO and 0.3385 by VIM). It is also 

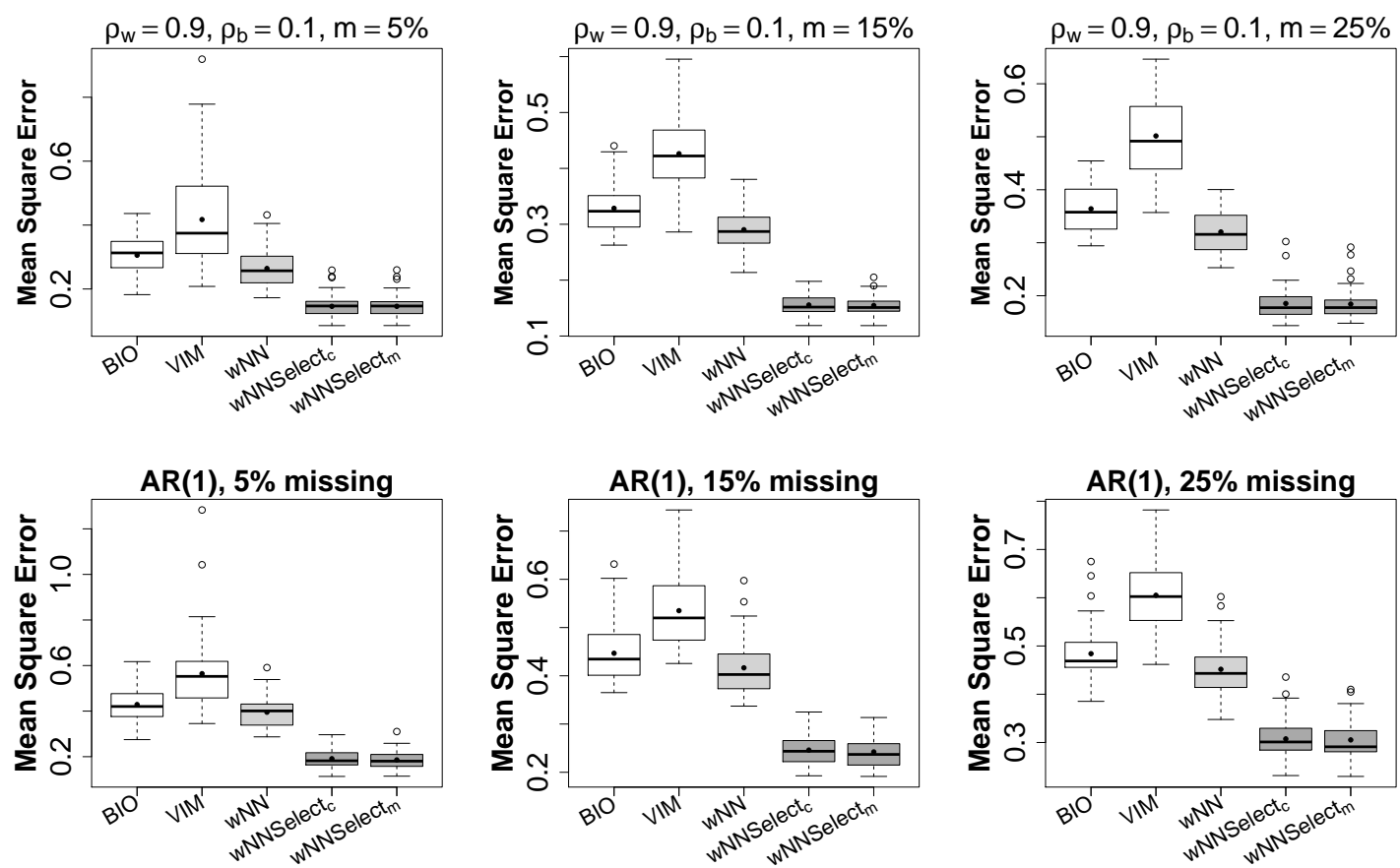

Figure 5: Comparison of $w N N$ Select (dark grey boxes), $w N N$ (light grey boxes) imputation using $L_{2}$ metric, and two benchmarks (white boxes); impute from Bioconductor (shown as BIO) \& VIM (shown as VIM) using Blockwise (upper panel) and Autoregressive (lower panel) correlation structure

obvious that the selection of dimension ( $w N N$ Select) reduces the MSEs when only the relevant information from selected predictors is used. Similar results are obtained for $n=100$ (Table 2: lower left panel). For the blockwise structure with $1 \%$ to $20 \%$ missing data there is not much difference concerning the alternative convex function used in the weighting procedure. But when percentage of the missing data is greater than $20 \%$ of the data matrix, the weighted function $\left(w N N\right.$ Delect $\left._{m}\right)$ with the power function (9) shows the best MSEs irrespective of the correlation structure.

In the case of autoregressive correlation with $n=50$ (Table 2: upper right panel), weighted selection of predictors with convex function (9), namely $w V$ VSelect $_{m}$, using $L_{2}$ metric provides smaller MSE (e.g., 0.1580 for $1 \%$ missing values and 0.3416 for $30 \%$ missing). Both of these values are much smaller than obtained by $w N N$ and benchmark imputation methods. Moreover, the best MSE is obtained by weighted $L_{2}$ with selection of predictors using convex function (9), namely $w N N$ Select $_{m}$. Similar results are found for $n=100$ (Table 2: lower right panel).

A comparative view of the performance for different levels of missing percentages for blockwise (lower panel) and autoressive (upper panel) is shown in Figure 6. To make them comparable all the plots are drawn on the same scale. It is seen that the mean squared errors get larger with increasing percentage of missing values. But the curve is more flatter when the data is from blockwise correlation. Overall, the proposed weighting functions distinctly outperform the available methods in all the data settings, in particular the $w_{N N S e l e c t}$ and $w N N$ Select $_{m}$ methods showed superior performance. 


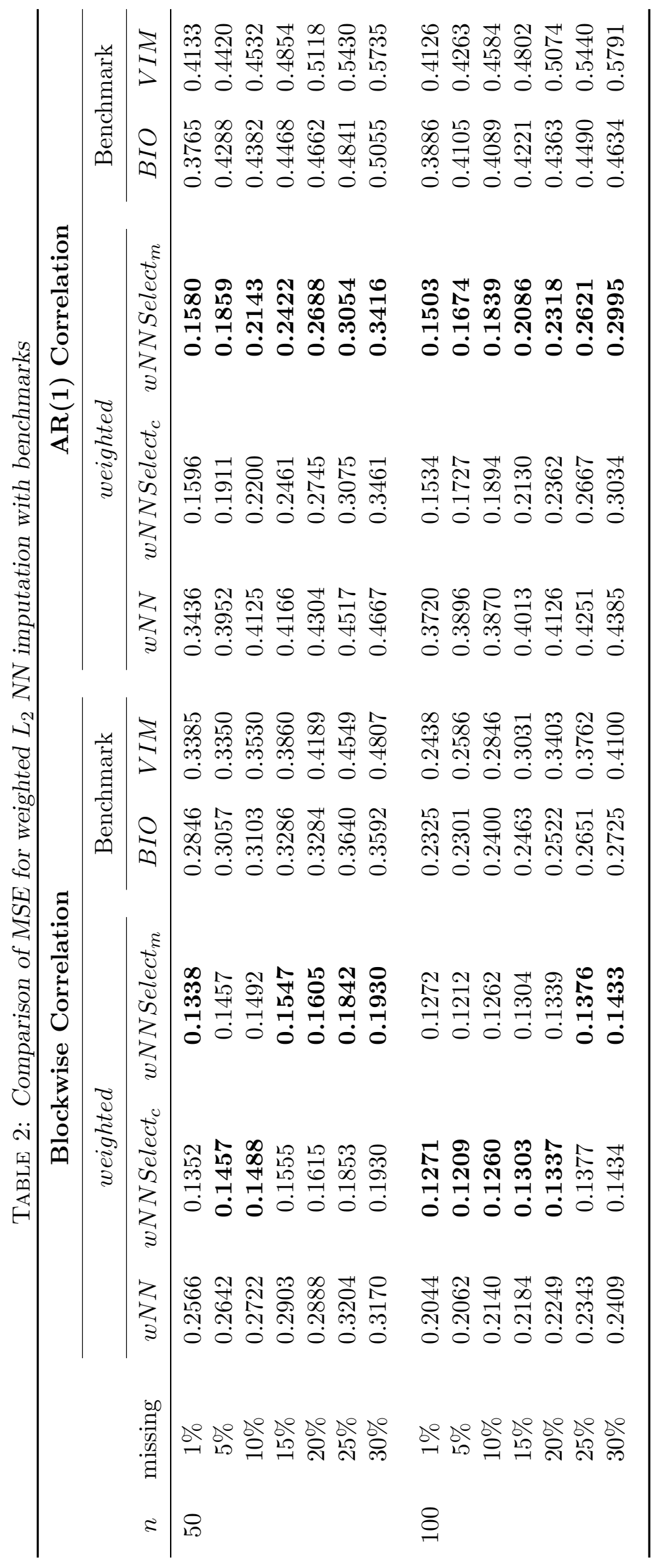



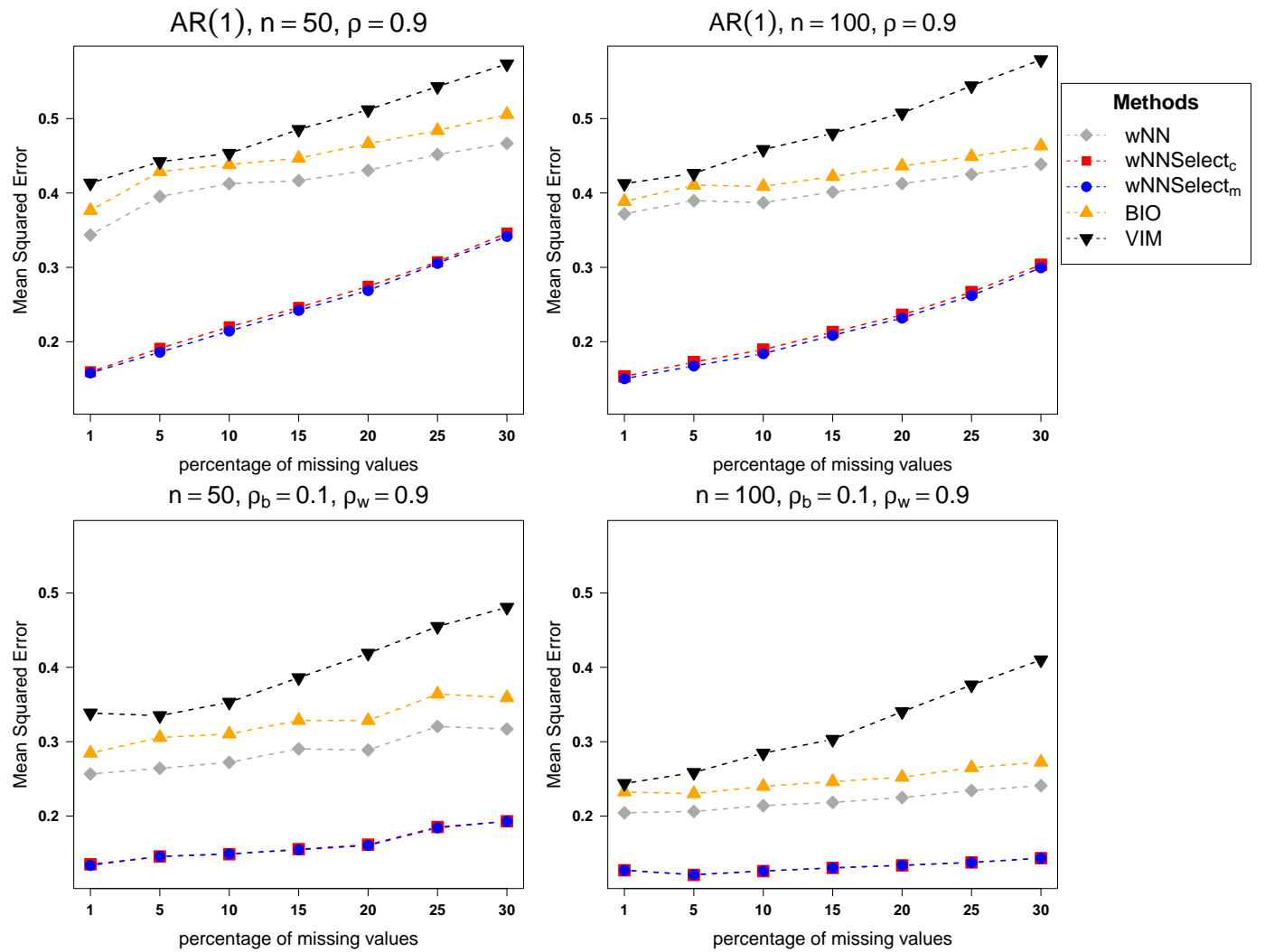

Figure 6: Comparison of $w N N$ Select at different percentages of missing data in autoregressive (upper panel) and blockwise (lower panel) structure. The tuning parameters $(\lambda, c)$ and $(\lambda, m)$ selected by cross-validation.

\section{Case Studies}

In this section we use several real data sets to compare imputation methods. We use two data sets from genetics and two data sets from non-genetic studies. The complete data sets without any missing values were used so that we can compare the results.

\subsection{Gene Expression Data}

In particular, gene expression data often contain missing values. Some previous studies on imputation of missing values in gene expression data are by Brock et al. (2008), Wasito and Mirkin (2005), Troyanskaya et al. (2003), Nguyen et al. (2004), Hastie et al. (1999), and Dudoit et al. (2002). We used subsets of gene expression data on two different types of human tumor namely lymphomas and leukemia. These data sets can be downloaded from the website http://www.gems-system.org/.

The data sets were preprocessed before using for imputation. A significance analysis was carried out to identify differentially expressed genes using samr package in R (R Core Team (2013)). The selected genes were standardized to normal scores. 


\section{DLBCL Data}

The gene expression data on Lymphomas was collected from 77 patients on 6,817 genes. The response was Diffuse large B-cell lymphomas (DLBCL) with $n=58$ patients or follicular lymphomas (FL) with $n=19$ patients. We used in our analysis the first 100 significant genes (variables) and all 77 patients.

\section{Leukemia Data}

The second data we use is based on three types of leukemia; Acute myelogenous leukemia (AML), acute lympboblastic leukemia (ALL) B-cell, and ALL T-cell. The complete data consists of the gene expression on 5,328 variables of 72 patients. The data for all 72 patients for first 100 significant genes was used.

\subsection{Non-Gene Expression Data}

\section{LSVT Voice Rehabilitation Data Set}

The LVST (Lee Silverman Voice Treatment) Global, a company specialising in voice rehabilitation assists the people with Parkinson's disease (PD). The data set was originally collected to determine the most parsimonious feature subset which helps to predict the binary response. The data are composed of a range of biomedical speech signal processing algorithms from 14 people who have been diagnosed with Parkinson's disease undergoing LSVT. The original study used 310 algorithms (predictors) to characterize 126 speech signals (samples). The response variable is binary, acceptable vs unacceptable phonation during rehabilitation. More information on data can be found in Tsanas et al. (2013). The data can be downloaded from the UCI Machine Learning Repository.

\section{LIBRAS Movement Database}

The data set contains 15 classes of 24 instances each, where each class references to a hand movement type in LIBRAS. The hand movement is represented as a bidimensional curve performed by the hand in a period of time. The curves were obtained from videos of hand movements, with the Libras performance from 4 different people, during 2 sessions. Each video corresponds to only one hand movement and has about 7 seconds. The total data consists of 360 instances of 90 numeric attributes. More information on data can be found in Dias et al. (2009). The data set is available on the UCI Machine Learning Repository.

All the variables were standardized before processing. The NN imputation techniques were applied to these four data sets by artificially setting $5 \%$ of the observations as missing completely at random (MCAR). The missing values were imputed by using weighted nearest neighbors with selection of variables ( $w N N$ Select) with the tuning parameters chosen by cross-validation. Also for the benchmark methods, the number of nearest neighbors was selected by crossvalidation. The procedure was repeated 10 times for all methods. The resulting averaged MSEs are shown in Table 3. The smallest MSE for each data set is shown in boldface.

In all these case studies, the minimum value of average MSE was obtained by one of the new imputation methods. For DLBCL data, the minimum, 0.5993, was obtained for the $L_{1}$ metric with the convex function (9) shown as $w N N$ Select $_{m}$. The the impute function in Bioconductor package gave the MSE of 0.72679 , and the VIM procedure had the highest value of MSE, 0.7889. 
TABLE 3: Average MSE results from real data sets

\begin{tabular}{|c|c|c|c|c|c|c|}
\hline \multirow[b]{2}{*}{ DATA } & \multicolumn{2}{|c|}{$L_{1}$ metric } & \multicolumn{2}{|c|}{$L_{2}$ metric } & \multicolumn{2}{|c|}{ Benchmark } \\
\hline & $w N N$ Select $_{c}$ & $w N N$ Select ${ }_{m}$ & $w N N$ Select $_{c}$ & $w N N$ Select $t_{m}$ & BIO & VIM \\
\hline DLBCL & 0.6424 & 0.5993 & 0.6504 & 0.6078 & 0.7267 & 0.7889 \\
\hline Leukemia & 0.7620 & 0.7461 & 0.7620 & 0.7292 & 0.8709 & 0.8272 \\
\hline LVST & 0.4401 & 0.4427 & 0.4142 & 0.4087 & 0.5821 & 0.5731 \\
\hline LIBRAS & 0.0799 & 0.0752 & 0.0353 & 0.0350 & 0.2903 & 0.3964 \\
\hline
\end{tabular}

Similar results are found for the leukemia data with 0.7279 as minimal average MSE from $w N N$ Select $t_{m}$ using $L_{2}$ metric. For LVST and LIBRAS movement data sets, the smallest MSE is obtained from $w N N$ Select ${ }_{m}$ using $L_{2}$ metric. It is obvious from table 3 that the proposed imputation procedure yields better results than the Bioconductor and the VIM packages in all the data sets.

\section{Concluding Remarks}

The main objective of this study was to introduce an improved nearest neighbor procedure for the imputation of missing values. The simulation results show that the proposed weighted imputation estimate performs better than the fixed or the un-weighted approach. We also compared $L_{1}$ and $L_{2}$ metrics in the weighted imputation of missing data and found $L_{2}$ metric to be slightly better than $L_{1}$ metric. The use of kernel functions for the computation of weights decreases the imputation error. Simulation results (not presented here) suggest that the Gaussian kernel provides smaller MSEs than other kernel functions.

To cope with the problem of high dimensional data, we proposed an imputation method with a weighted selection of predictors. The procedure uses cross-validation for optimal selection of the tuning parameters. In particular, for highly correlated data, the proposed NN imputation procedure shows promising results, also in the case of a high proportion of missing data. The simulation studies as well as real data sets confirm these results.

\section{References}

Allison, P. D. (2001). Missing data. Number 136. Sage.

Atkeson, C., A. Moore, and S. Schaal (1997). Locally weighted learning. Artificial Intelligence Review 11(1-5), 11-73.

Batista, G. E. and M. C. Monard (2002). A study of k-nearest neighbour as an imputation method. HIS 87, 251-260.

Bø, T. H., B. Dysvik, and I. Jonassen (2004). Lsimpute: accurate estimation of missing values in microarray data with least squares methods. Nucleic Acids Research 32(3), e34-e34.

Brock, G. N., J. R. Shaffer, R. E. Blakesley, M. J. Lotz, and G. C. Tseng (2008). Which missing value imputation method to use in expression profiles: a comparative study and two selection schemes. BMC Bioinformatics 9(1), 12. 
Cai, Z., M. Heydari, and G. Lin (2006). Iterated local least squares microarray missing value imputation. Journal of Bioinformatics and Computational Biology 4 (05), 935-957.

Dias, D. B., R. C. Madeo, T. Rocha, H. H. Biscaro, and S. M. Peres (2009). Hand movement recognition for brazilian sign language: a study using distancebased neural networks. In Neural Networks, 2009. IJCNN 2009. International Joint Conference on, pp. 697-704. IEEE.

Dudoit, S., J. Fridlyand, and T. P. Speed (2002). Comparison of discrimination methods for the classification of tumors using gene expression data. Journal of the American Statistical Association 97, 77-87.

Eskelson, B. N., H. Temesgen, V. Lemay, T. M. Barrett, N. L. Crookston, and A. T. Hudak (2009). The roles of nearest neighbor methods in imputing missing data in forest inventory and monitoring databases. Scandinavian Journal of Forest Research 24(3), 235-246.

Feten, G., T. Almoy, and A. H. Aastveit (2005). Prediction of missing values in microarray and use of mixed models to evaluate the predictors. Statistical Applications in Genetics and Molecular Biology 4(1), 10.

Fix, E. and J. L. Hodges (1951). Discriminatory analysis-nonparametric discrimination: consistency properties. Technical report, DTIC Document.

Gower, J. C. (1971). A general coefficient of similarity and some of its properties. Biometrics, 857-871.

Hastie, T., R. Tibshirani, B. Narasimhan, and G. Chu (2013). impute: impute: Imputation for microarray data. http://www.bioconductor.org/packages/ release/bioc/html/impute.html. R package version 1.36.0.

Hastie, T., R. Tibshirani, G. Sherlock, M. Eisen, P. Brown, and D. Botstein (1999). Imputing missing data for gene expression arrays.

Hron, K., M. Templ, and P. Filzmoser (2010). Imputation of missing values for compositional data using classical and robust methods. Computational Statistics and Data Analysis 54(12), 3095-3107.

Hudak, A. T., N. L. Crookston, J. S. Evans, D. E. Hall, and M. J. Falkowski (2008). Nearest neighbor imputation of species-level, plot-scale forest structure attributes from lidar data. Remote Sensing of Environment 112(5), $2232-2245$.

Johansson, P. and J. Hakkinen (2006). Improving missing value imputation of microarray data by using spot quality weights. BMC Bioinformatics 7(1), 306.

Junninen, H., H. Niska, K. Tuppurainen, J. Ruuskanen, and M. Kolehmainen (2004). Methods for imputation of missing values in air quality data sets. Atmospheric Environment 38(18), 2895-2907.

Kim, H., G. H. Golub, and H. Park (2005). Missing value estimation for dna microarray gene expression data: local least squares imputation. Bioinformatics $21(2), 187-198$. 
Kim, K.-Y., B.-J. Kim, and G.-S. Yi (2004). Reuse of imputed data in microarray analysis increases imputation efficiency. BMC Bioinformatics 5(1), 160.

Liew, A. W.-C., N.-F. Law, and H. Yan (2011). Missing value imputation for gene expression data: computational techniques to recover missing data from available information. Briefings in Bioinformatics 12(5), 498-513.

Little, R. J. and D. B. Rubin (1987). Statistical analysis with missing data, Volume 539. Wiley New York.

Malarvizhi, M. R. and D. A. S. Thanamani (2012). K-nearest neighbor in missing data imputation. International Journal of Engineering Research and Development 5.

Moorthy, K., M. Saberi Mohamad, and S. Deris (2014). A review on missing value imputation algorithms for microarray gene expression data. Current Bioinformatics 9(1), 18-22.

Myrtveit, I., E. Stensrud, and U. H. Olsson (2001). Analyzing data sets with missing data: an empirical evaluation of imputation methods and likelihoodbased methods. Software Engineering, IEEE Transactions on 27(11), 9991013 .

Nguyen, D. V., N. Wang, and R. J. Carroll (2004). Evaluation of missing value estimation for microarray data. Journal of Data Science 2(4), 347-370.

Ouyang, M., W. J. Welsh, and P. Georgopoulos (2004). Gaussian mixture clustering and imputation of microarray data. Bioinformatics 20(6), 917-923.

R Core Team (2013). R: A language and environment for statistical computing. http://www.R-project.org/.

Schafer, J. L. (2010). Analysis of incomplete multivariate data. CRC press.

Scheel, I., M. Aldrin, I. K. Glad, R. SÃ,rum, H. Lyng, and A. Frigessi (2005). The influence of missing value imputation on detection of differentially expressed genes from microarray data. Bioinformatics 21(23), 4272-4279.

Sehgal, M. S. B., I. Gondal, and L. S. Dooley (2005). Collateral missing value imputation: a new robust missing value estimation algorithm for microarray data. Bioinformatics 21(10), 2417-2423.

Templ, M., A. Alfons, A. Kowarik, and B. Prantner (2013). Vim: Visualization and imputation of missing values. http://CRAN.R-project.org/package= VIM. R package version 4.0.0.

Troyanskaya, O., D. Botstein, and R. Altman (2003). Missing value estimation. In D. Berrar, W. Dubitzky, and M. Granzow (Eds.), A Practical Approach to Microarray Data Analysis, pp. 65-75. Springer US.

Troyanskaya, O., M. Cantor, G. Sherlock, P. Brown, T. Hastie, R. Tibshirani, D. Botstein, and R. B. Altman (2001). Missing value estimation methods for dna microarrays. Bioinformatics 17(6), 520-525.

Tsanas, A., M. A. Little, C. Fox, and L. O. Ramig (2013). Objective automatic assessment of rehabilitative speech treatment in parkinson's disease. 
Waljee, A. K., A. Mukherjee, A. G. Singal, Y. Zhang, J. Warren, U. Balis, J. Marrero, J. Zhu, and P. D. Higgins (2013). Comparison of imputation methods for missing laboratory data in medicine. BMJ Open 3(8).

Wasito, I. and B. Mirkin (2005). Nearest neighbour approach in the least-squares data imputation algorithms. Information Sciences 169(1), 1-25.

Wong, J. (2013). imputation: imputation. http://CRAN.R-project.org/ package=imputation. $\mathrm{R}$ package version 2.0.1.

Yoon, D., E.-K. Lee, and T. Park (2007). Robust imputation method for missing values in microarray data. BMC Bioinformatics 8(Suppl 2), S6.

Zhang, X., X. Song, H. Wang, and H. Zhang (2008). Sequential local least squares imputation estimating missing value of microarray data. Computers in Biology and Medicine 38(10), 1112-1120. 